\title{
EDITORIAL
}

\section{Editorial: Reproducible Research in Medical Imaging}

\author{
Kingshuk Roy Choudhury, ${ }^{1}$ Ray Gibson ${ }^{2}$ \\ ${ }^{1}$ Department of Radiology, Duke University, Suite 302, Hock Plaza 2424 Erwin Road, Durham, NC 27705, USA \\ ${ }^{2}$ Molecular Imaging and Biology, Holland, PA, USA
}

$\mathrm{T}$ he American Physical Society defines science as: "Science is the systematic enterprise of gathering knowledge about the universe and organizing and condensing that knowledge into testable laws and theories." It goes on to note that: "The success and credibility of science are anchored in the willingness of scientists to expose their ideas and results to independent testing and replication by other scientists. This requires the complete and open exchange of data, procedures, and materials [1]". As a corollary of this definition, one could argue that ideally, anything published in a scientific paper should be reproducible by the reader [2]. However, increasing sophistication, expense, and inaccessibility to subjects, such as test animals in preclinical studies and patients in clinical studies, precludes most readers from performing the work described in typical papers on current biomedical research. The only aspects of analysis that are practically reproducible are those involving computation and data analysis. Even in this limited context, page pressure in print journals means that most modern scientific papers do not carry enough detail for the reader to be able to reproduce the reported research.

In the field of clinical research, many journals have adopted a set of minimum standards for reporting common types of studies such as randomized control trials (CONSORT [3]) and meta-analysis or reviews (PRISMA [4]). Of particular relevance to the medical imaging community is the STARD initiative [5] for reporting diagnostic accuracy studies. These standards typically constitute a checklist of some 20-30 items, under headings such: Participants, Test methods, Statistical methods, Test results, Estimates, etc. An example of how adherence to these standards helps reproducible research was recently seen in a letter to this journal [6] pointing out an error in sensitivity calculations in [7]. To the authors' credit, the error could be spotted because a cross-classified table of positive and negative findings was published in [7], as recommended by item 19 on the STARD checklist. If only the sensitivity values had been reported, this error would have gone unnoticed.

Correspondence to: Kingshuk Roy Choudhury; e-mail: kingshuk@duke.edu
In a field such as molecular imaging, the level of sophistication of many published analyses is such that it would be hard to verify the research by visual inspection alone. Apart from novelty in the data, many papers present computational or analysis methodology that is novel to all except a few readers. However, sophistication in itself is no guarantee of correctness. A series of papers on a flawed gene-expression test, published in top scientific journals by a cancer research group at Duke, have since been retracted (en.wikipedia.org/wiki/Anil_Potti). In their retraction, the authors state: "We wish to retract this article because we have been unable to reproduce certain key experiments described in the paper regarding validation and use of the colon cancer prognostic signature" [8]. It might seem too much to expect readers to be able to reproduce such research by reading these papers. Reassuringly, there is a straightforward solution to this problem. Researchers in numerically sophisticated fields such as signal processing [9] and scientific computing [10] and bioinformatics [11] have demonstrated how it is possible to give readers access to archived computer code, written in modern programming environments such as Matlab, Python, and R, used to generate the figures and tables in these papers. Anyone with a computer which has the appropriate software loaded should be able copy and paste to achieve the same results. Such code (together with descriptions as necessary) can be included as supplementary material to the published article either on the journal or the author's own website, whichever is permanently available. In the field of image analysis, a by-product of archiving computational procedures is that it will address questions of subjectivity that perennially arise when thresholding images or drawing regions of interest.

Potentially more complicated than sharing computational code is the issue of sharing data, particularly raw data from clinical imaging studies. This data can be difficult and expensive to acquire, be very large in size, sometimes saved in proprietary formats and contain private health information. But all of these problems can technically be overcome. In recent years, we have seen the rise of a number of large publicly available imaging databases, such as the Alzheimer's Disease Neuroimaging Initiative [12] and the Lung Image Database Consortium [13]. On a related note, data (and code) sharing is a required consideration of all research funding applications in genomics research [14]. Genomic 
data share all of the problems listed above for imaging data. Most genome sequences on which research has been published are publicly available over the internet through GenBank [15] and sister websites around the world. It is worth noting that the Duke cancer group had shared their data and software in their publications. Discrepancies in their results were first noticed by a group who tried to reproduce their results, but failed [16]. The NIH should give serious consideration to creating an equivalent "ImageBank" which will enable data (and code) publishing to become routine for medical imaging research.

Apart from contributing to progress of science in general, reproducible research can have certain tangible benefits to the authors themselves. It is a fact of life that graduate students and postdocs move on, taking their sometimes unique knowledge and expertise with them. Archiving their work as reproducible research would make it painless for their successors and/or erstwhile supervisors to progress from the point where they left off. Another unanticipated benefit that has been documented is an increase in the citation rate for published papers that share research data [17]. The following words, which appear in the conclusion of the Institute of Medicine report [18] on the flawed gene-expression tests at Duke, are particularly relevant: "These guidelines, if adopted, can ensure that progress in omics-based test development is grounded in sound scientific practice and is reproducible, resulting not only in improved health care but also - and perhaps equally important-in continued public trust." Introduction of such guidelines for reproducibility into all areas of biomedical research is a must, particularly in medical imaging, because the results of imaging studies can ultimately influence patient treatment and outcome.

\section{References}

1. Ethics and values: "What is science?" http://www.aps.org/policy/ statements/99 6.cfm. Accessed 9 June 2012
2. An sweave demo; Literate programming in $\mathrm{R}$; reproducible research. http://users.stat.umn.edu/ geyer/Sweave/. Accessed 9 June 2012

3. CONSORT: Consolidated Standards of Reporting Trials. http:// www.consort-statement.org/. Accessed 9 June 2012

4. Preferred reporting items for systematic reviews and meta-analyses: the prisma statement. http://www.prisma-statement.org/. Accessed 9 June 2012

5. Standards for the reporting of diagnostic accuracy studies. http:// www.stard-statement.org/. Accessed 9 June 2012

6. White LR et al (2011) Comparison of CT, PET, and PET/CT for staging of patients with indolent non-hodgkin's lymphoma: statistical errors in Fueger et al. (2009). Mol Imaging Biol. doi:10.1007/ s11307-011-0513-3

7. Fueger BJ, Yeom K, Czernin J et al (2009) Comparison of CT, PET, and PET/CT for staging of patients with indolent non-Hodgkin's lymphoma. Mol Imaging Biol 11:269-274

8. Garman KS, Acharya CR, Edelman E et al (2011) Retraction for Garman et al., A genomic approach to colon cancer risk stratification yields biologic insights into therapeutic opportunities. Proc Nat Acad Sci 108:17569

9. Vandewalle P, Barrenetxea G, Jovanovic I et al (2007) Experiences with reproducible research in various facets of signal processing research. Proc IEEE Intl Conf Acoust Speech Signal Proc 4:12531256

10. Leveque R (2009) Python tools for reproducible research on hyperbolic problems. Comput Sci Eng 11:19-27

11. Gentleman R (2004) Reproducible research: a bioinformatics case study. Statist Appl Genet Molec Biol 4. doi:10.2202/15446115.1034

12. Alzheimer's disease neuroimaging initiative [http://adni.loni.ucla.edu/]. Accessed 9 June 2012

13. Lung image database consortium [http://imaging.cancer.gov/program sandresources/informationsystems/lidc]. Accessed 9 June 2012

14. Kaye J, Heeney C, Hawkins N et al (2009) Data sharing in genomicsre-shaping scientific practice. Nat Rev Genet 10:331-335

15. GenBank [http://www.ncbi.nlm.nih.gov/genbank/]. Accessed 9 June 2012

16. Coombes KR, Wang J, Baggerly KA (2007) Microarrays: retracing steps. Nat Med 13:1276-1277, author reply 1277-1278

17. Piwowar H, Day R, Fridsma D (2007) Sharing detailed research data is associated with increased citation rate. PLoS One 2:e308

18. Omenn GS et al (2012) Evolution of translational omics: lessons learned and the path forward. Institute of Medicine of the National Academies, Washington DC 